\title{
Technologies in Diabetes-the Tenth ATTD Yearbook
}

\author{
Tadej Battelino, $\mathrm{MD}^{1,2}$ and Moshe Phillip, $\mathrm{MD}^{3,4}$
}

A FTER THE FESTIVE INTRODUCTION TO last year's Advanced Technologies and Treatments for Diabetes (ATTD) Yearbook, when we celebrated the inauguration of (hybrid) closed-loop insulin delivery into everyday clinical reality in the United States, we had to face, as usual in life, the fact that until diabetes is finally cured, we will still have to deal with multiple ongoing challenges. The overwhelming evidence of benefits conveyed by the regular use of continuous glucose monitoring (CGM) highlighted the incomprehensible gap between research and routine care practices. CGM is - surprisingly to many of us - still not the predominant treatment modality despite the overwhelming evidence, mainly because public reimbursement systems in many wealthy Western societies still spend millions of dollars on chronic complications of diabetes rather than investing in better care with the regular use of CGM, thus preventing them.

"There is nothing new under the sun" as the ancient proverb says. It took decades before famous diabetes professors accepted the benefit of self-monitoring of blood glucose. However, the frustration and anger of people with diabetes (PWDs) seems understandable and legitimate. Intermittent (Flash) glucose monitoring made it into several reimbursement systems with lowered prices and public pressure, alleviating some of the imminent need of PWDs. However, the exigent need for more comprehensive reimbursement for diabetes care remains colossal.

Imagine if a majority of PWDs could get CGM reimbursed and could keep their glucose concentration within range most of the time. Time in range is the new reality, a solid clinical outcome to erudite diabetologists and, unfortunately, a mere gimmick to those firmly rooted in denial regarding the damage glucose concentrations outside the near-normal range cause to several organs. The attention that developed societies have recently begun to focus on the human brain seems timely and fundamental also for PWDs. Hyperglycemia and proportion of time above range was firmly associated with damage to the brain in young, adult, and aging populations of PWDs. Cognitive impairment and early dementia may soon represent one of the biggest burdens on our aging societies, both in social and fiscal dimensions. CGM can therefore become a cost-saving treatment modality-just imagine!

Clinicians, diabetes care providers, researchers, industries, investors, reimbursement authorities, and recently most importantly PWDs convene at ATTD annual meetings to infuse some optimism and fairness into the potential novel technologies and treatments for diabetes could bring to many. This ATTD 2018 Yearbook again strives to provide some firm evidence and clarity through the eyes of our expert associate editors and authors of the 13 articles. Please join us in amazement at the wealth of data and insight this ATTD 2018 Yearbook brings to us: diabetes technologies and novel therapies help improve diabetes outcomes ever better and more. That said, PWDs need universal access to and sustained reimbursement of all these proven-efficient therapies.

Our continuous dream has sprouted a new branch: that all people with diabetes might have access to the evidence-based novel treatment modalities. Joined with ATTD, we managed to foster and accelerate research, innovation, and investment into advanced treatments for diabetes; let us now make them available to all who are in obvious need.

Last, but not least, we wish to extend our heartfelt gratitude to our coauthors for sharing their knowledge, expertise, and valuable time in writing their articles in this ATTD Yearbook.

\footnotetext{
${ }^{1}$ University Medical Center-University Children's Hospital Ljubljana, Ljubljana, Slovenia.

${ }^{2}$ Faculty of Medicine, University of Ljubljana, Ljubljana, Slovenia.

${ }^{3}$ Jesse Z and Sara Lea Shafer Institute for Endocrinology and Diabetes, Schneider Childresn's Medical Center of Israel, Petah Tikva, Israel.

${ }^{4}$ Sackler Faculty of Medicine, Tel Aviv University, Tel Aviv, Israel.
} 